\title{
The Large-Angle Photon Veto System for the NA62 Experiment at CERN
}

\section{Paolo Massarotti*}

Dipartimento di Scienze Fisiche dell'Università degli studi di Napoli "Federico II" and Sezione INFN, Napoli, Italy massarotti@na. infn. it

\section{F. Ambrosino, D. Di Filippo, M. Napolitano, G. Saracino}

Dipartimento di Scienze Fisiche dell'Università degli studi di Napoli "Federico II" and Sezione INFN, Napoli, Italy

\author{
B. Angelucci, F. Costantini, R. Fantechi, S. Gallorini, S. Giudici, I. Mannelli, \\ F. Rafaelli, and S. Venditti \\ Dipartimento di Fisica dell'Università and Sezione INFN, Pisa, Italy.
}

\section{A. Antonelli, M. Moulson, M. Raggi, and T. Spadaro}

Laboratori Nazionali di Frascati dell'INFN, Frascati, Italy.

G. D’Agostini, E. Leonardi, V. Palladino, M. Serra, and P. Valente

Dipartimento di Fisica dell'Università “La Sapienza” and Sezione INFN, Roma, Italy.

\begin{abstract}
The branching ratio (BR) for the decay $K^{+} \rightarrow \pi^{+} v \bar{v}$ is a sensitive probe for new physics. The NA62 experiment at the CERN SPS will measure this BR to within about $10 \%$. To reject the dominant background from channels with final state photons, the large-angle vetoes (LAVs) must detect photons of energy as low as $200 \mathrm{MeV}$ with an inefficiency of less than $10^{-4}$, as well as provide energy and time measurements with resolutions of $10 \%$ and $1 \mathrm{~ns}$ for $1 \mathrm{GeV}$ photons. The LAV detectors make creative reuse of lead glass blocks recycled from the OPAL electromagnetic calorimeter barrel. We describe the mechanical design and challenges faced during construction, the characterization of the lead glass blocks and the development of front-end electronics to allow simultaneous time and energy measurements over an extended dynamic range using the timeover-threshold technique.
\end{abstract}

36th International Conference on High Energy Physics,

July 4-11, 2012

Melbourne, Australia

\footnotetext{
*Speaker.
} 


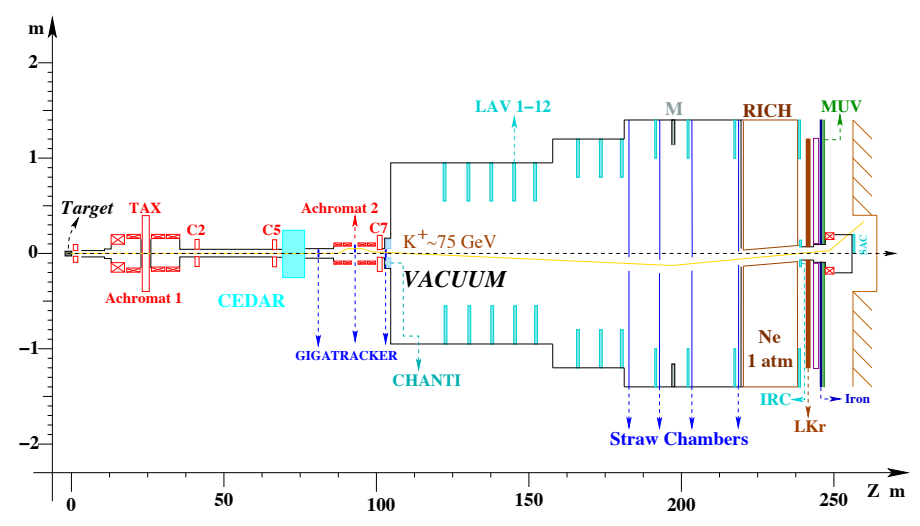

Figure 1: The NA62 experimental layout.

\section{The NA62 Experiment}

The decays $K^{+} \rightarrow \pi^{+} v \bar{v}$ and $K_{L} \rightarrow \pi^{0} v \bar{v}$ are flavor-changing neutral-current processes whose amplitudes are dominated by $Z$-penguin and box diagrams. Because there are no contributions from long-distance processes with intermediate photons and because the hadronic matrix elements can be obtained from rate and form factor measurements for common $K \rightarrow \pi \ell v_{\ell}$ decays, the branching ratios (BRs) for the $K \rightarrow \pi v \bar{v}$ decays can be calculated in the Standard Model (SM) with minimal intrinsic uncertainty (see [1] for a recent review). The BRs for these decays are therefore a sensitive probe of the SM flavor sector and provide constraints on the CKM unitarity triangle that are complementary to those from measurements of $B$-meson decays.

On the other hand, the tiny BRs for these decays are notoriously difficult to measure, not least because of the three-body kinematics with two undetectable neutrinos in the final state. At present, the experimental value of the BR for the decay $K^{+} \rightarrow \pi^{+} v \bar{v}$ is $1.73_{-1.05}^{+1.15} \times 10^{-10}$ on the basis of seven detected candidate events [2]. The goal of NA62, an experiment at the CERN SPS, is to detect $\sim 100 K^{+} \rightarrow \pi^{+} v \bar{v}$ decays with a S/B ratio of 10:1 in two years of data taking beginning in 2014. The experiment is fully described in [3]. The experimental layout is illustrated in 1 .

NA62 will make use of a 75-GeV unseparated positive secondary beam with a total rate of nearly $800 \mathrm{MHz}$, of which $\sim 50 \mathrm{MHz}$ is $K^{+}$'s. The $K^{+}$'s are identified by the CEDAR differential Cerenkov counter in the beamline. All $800 \mathrm{MHz}$ of beam particles are tracked by three siliconpixel tracking detectors (the Gigatracker) located at the achromat just upstream of the vacuum decay volume, providing event-by-event measurements of the $K^{+}$trajectory and momentum. The decay volume is evacuated to $10^{-6}$ mbar in order to minimize background between interactions and residual gases. It begins $\sim 100 \mathrm{~m}$ downstream of the production target, is $\sim 110 \mathrm{~m} \mathrm{long}$, and consists of segments of increasing diameter, from $\sim 2 \mathrm{~m}$ upstream to $\sim 3 \mathrm{~m}$ downstream. $5 \mathrm{MHz}$ of kaon decays are observed in the 65-m fiducial decay region, in the upstream part of the vacuum tank. The ring-shaped large-angle photon vetoes (LAVs) are placed at 12 stations along the vacuum volume and provide full coverage for decay photons with $8.5 \mathrm{mrad}<\theta<50 \mathrm{mrad}$.

Assuming an acceptance for signal events of about $10 \%$, the experiment must be able to reject background from the dominant $K^{+}$decays such as $K^{+} \rightarrow \pi^{+} \pi^{0}$ at the level of $10^{12}$. Kinematic cuts on the $K^{+}$and $\pi^{+}$tracks (as reconstructed in the Gigatracker and straw chambers, respec- 

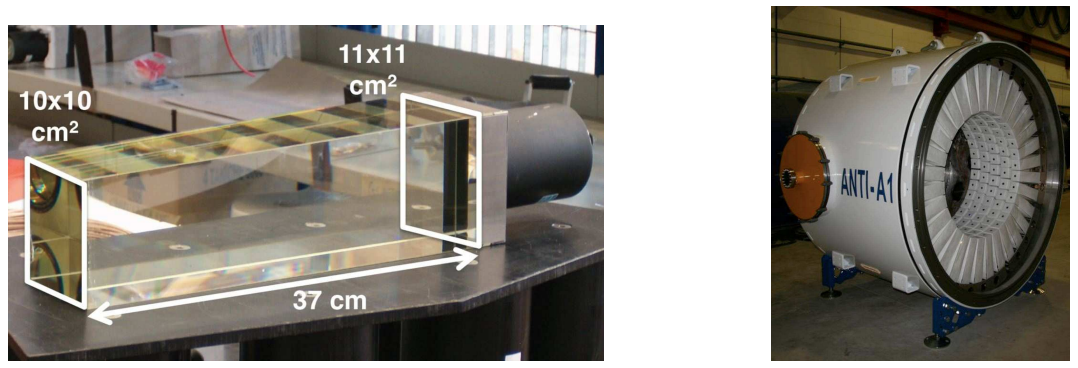

Figure 2: Left: A module from the OPAL calorimeter, without wrapping and with reinforcement plates at the interface between the glass and the steel flange. Right: completed prototype A1 veto station making use of the OPAL lead glass calorimeter elements.

tively) provide a rejection factor of $10^{4}$ and ensure that the photons from the $\pi^{0}$ have $40 \mathrm{GeV}$ of energy.Photons from $K^{+} \rightarrow \pi^{+} \pi^{0}$ intercepted by the LAVs may have energies from a few tens of $\mathrm{MeV}$ to several $\mathrm{GeV}$. In order to detect the $\pi^{0}$ with an inefficiency of $\leq 10^{-8}$, the maximum tolerable inefficiency in the LAV detectors for photons with energies as low as $200 \mathrm{MeV}$ is $10^{-4}$. In addition, the LAV detectors must have good time resolution $(\sim 1 \mathrm{~ns})$ to allow signals from incident particles to be identified with the correct event, and good energy resolution $(\sim 10 \%$ at 1 $\mathrm{GeV}$ ) for precise vetoing and use in full-event reconstruction. The system must also be sensitive to minimum-ionizing particles. Finally, the LAV detectors must be compatible with operation in a vacuum of $10^{-6}$ mbar.

\section{The Large-Angle Veto System}

The NA62 LAV detectors make creative reuse of lead glass blocks recycled from the OPAL electromagnetic calorimeter barrel [4], which became available in 2007, when various technologies were under consideration for the construction of the LAV detectors. A Prototype instruments was constructed, and tested with the electron beam at the Frascati Beam-Test Facility. These tests demonstrated that the inefficiency for the detection of single, tagged electrons with the OPAL lead glass modules was $1.2_{-0.8}^{+0.9} \times 10^{-4}$ at $203 \mathrm{MeV}$ and $1.1_{-0.7}^{+1.9} \times 10^{-5}$ at $483 \mathrm{MeV}$. Basing the construction of the LAV system on the OPAL lead glass modules provides significant economic advantages. The modules from the central part of the OPAL electromagnetic calorimeter barrel consist of blocks of Schott SF57 lead glass. This material has a radiation length equal to $X_{0}=1.50 \mathrm{~cm}$; its index of refraction is $n \approx 1.85$ at $\lambda=550 \mathrm{~nm}$ and $n \approx 1.91$ at $\lambda=400 \mathrm{~nm}$. Electromagnetic showers in the lead glass are detected by virtue of the Cerenkov light produced; our measurements indicate that, averaged over modules, minimum ionizing particles produce about 0.34 p.e. per $\mathrm{MeV}$ of deposited energy. Each block is read out at the back side by a Hamamatsu R2238 76-mm PMT, which is optically coupled via a 4-cm long cylindrical light guide of SF57 of the same diameter as the PMT. The rear face of the glass block is glued to a $1-\mathrm{cm}$ thick stainless steel flange featuring the holes that allow mounting hardware to be attached and a circular cutout for the light guide. A mu-metal shield surrounding the PMT and light guide is also glued to the flange. A complete module (block plus PMT) is a monolithic assembly; the block and PMT cannot be independently replaced. Figure 2 (left) shows a picture of a complete module. 
A LAV station is made by arranging these blocks around the inside of a segment of vacuum tank, with the blocks aligned radially to form an inward-facing ring. Multiple rings are used in each station in order to provide the desired depth for incident particles. The blocks in successive rings are staggered in azimuth; the rings are spaced longitudinally by about $1 \mathrm{~cm}$. The LAV system consists of a total of 12 stations, the diameter of which increases with distance from the target. For stations A1-A5, A6-A8, and A9-A11, apart from the different sizes and block configurations, the designs are conceptually similar. The geometry of A12 is not yet final; this station is operated in air and its design is different from that of the other stations. Since the spaces between the blocks are significantly smaller in the larger-diameter vessels, fewer layers are necessary. As a result of the staggering scheme, particles incident on any station are intercepted by blocks in at least three rings, for a total minimum effective depth of 21 radiation lengths. The vast majority of incident particles are intercepted by four or more blocks $\left(27 X_{0}\right)$.

\section{LAV Construction}

The LAV vacuum vessels are constructed from 25 -mm thick steel plate, rolled into a cylinder and arc welded along the seam. Structural steel (S275JR) is used for stations A1 to A8 and A11. Stations $A 9$ and $A 10$ are mounted astride the spectrometer magnet, so non-magnetic $\left(\mu / \mu_{0} \approx 1.01\right)$ 304L stainless steel is used for these stations. To maintain the design vacuum of $\sim 10^{-6}$ mbar, all components of the LAV detectors must be carefully cleaned. The original wrapping is removed and the blocks are thoroughly cleaned with acetone or isopropyl alcohol. They are then wrapped with a new laser-cut and heat-welded Tyvek cover. The blocks are tested and characterized 12 at a time using a test station featuring an LED pulser and cosmic-ray telescope. The PMT gains are measured first, by varying the intensity of the light pulses from the LEDs as well as the PMT HV settings and mapping out the response for each block. Using the gain curves so obtained, the PMTs are then set to a reference value of the gain $\left(9 \times 10^{5}\right.$ or $\left.1 \times 10^{6}\right)$ and the response to cosmic rays selected by the telescope is measured; the photoelectron yield for the block (p.e./MeV) is then obtained assuming that vertically incident cosmic rays leave $77 \mathrm{MeV}$ in each block. As noted above, photoelectron yields of 0.34 p.e./MeV are typical. Finally, using the gain curves and the measurements of photoelectron yield, the PMT voltages are set to the values expected to produce a common output charge level of $4.5 \mathrm{pC}$ for cosmic ray events. The response is measured and the HV setting is validated.

The OPAL design features an optical port at the base of each module. Blue LEDs are installed in these optical ports as part of the calibration and monitoring system, and will allow monitoring of the operational status and relative timing for each block. In principle, the LED system should allow in-situ gain measurement as well. After testing and characterization, the blocks are arranged in groups of four in an aluminum mounting bracket. For the installation, the vacuum vessel is turned on end. In order to preserve uniformity of signal shapes and timing across channels, all signal (and LED) cables have the same length.

\section{Front-End Electronics}

As noted above, the LAVs must furnish time and energy measurements over a large range of 


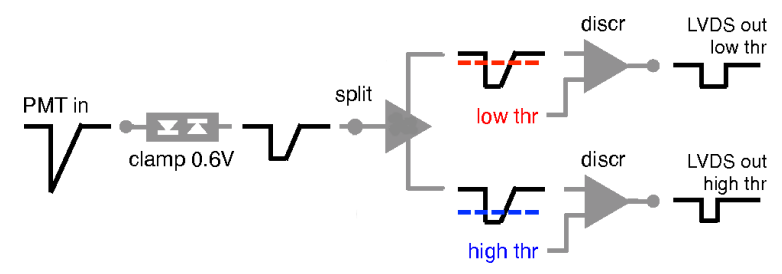

Figure 3: Conceptual schematic of the ToT discriminator board.

incident photon energies. For reasons of cost and simplicity, we have decided on a readout scheme based on the time-over-threshold (ToT) technique. This scheme is implemented using a dedicated front-end ToT discriminator board of our own design [6] and a digital readout board (TEL62) used by various NA62 detector subsystems [7]. The ToT discriminator converts the analog signals from the detector to low-voltage differential signal (LVDS) pulses, with width equal to the duration of the analog signal from the detector above a specified threshold. The signal from each PMT is compared to two different thresholds, a low threshold of about $5 \mathrm{mV}$ and a high threshold expected to be about $50 \mathrm{mV}$, corresponding to two different LVDS outputs. (For comparison, the expected noise level is $2 \mathrm{mV}$ under normal operating conditions.) The TEL62 is based on the design of the TELL1 readout board developed for the LHCb experiment. On the TEL62, TDC mezzanines measure the leading and trailing times of the LVDS pulses. Using a time-to-charge calibration parameterization, the FPGA on board the TEL62 calculates the time corrected for slewing, as well the charge for each hit as reconstructed from the pulse width above threshold. This information is sent to the subsequent DAQ stages. Level-0 trigger primitives are also calculated on board the TEL62 and sent to the level-0 trigger processor.

A conceptual schematic of the ToT discriminator board is presented in fig. 3 to illustrate the signal processing for a single channel. Since each channel is discriminated against two different thresholds, the board has 32 input channels and 64 output channels.

\section{Test-Beam Performance}

The A2 LAV station was tested using the positive secondary beam in the T9 area at the CERN PS in August 2010. Data were collected at various beam momenta over the interval 0.3-10 GeV. The composition of the $\mathrm{T} 9$ beam changes as a function of the momentum setting. At $0.3 \mathrm{GeV}$, the beam is roughly $70 \% e^{+}$and $30 \% \pi^{+}$(including decay $\mu^{+}$), while above $6 \mathrm{GeV}$ the $e^{+}$component drops off sharply and at $10 \mathrm{GeV}$ the beam is roughly a $50 \%$ mix of $\pi^{+} / \mu^{+}$and $p$. Two threshold Cerenkov counters in the beamline using $\mathrm{CO}_{2}$ at adjustable pressure allowed samples enriched $e^{+}$ and $\mu^{+}$to be selected. The T9 detectors also included two scintillation paddles and a beam wire chamber for beam counting and diagnostics. The beam focus was $2 \mathrm{~m}$ downstream of the beam wire chamber, and A2 was positioned so that the beam focus coincided approximately with the first layer of lead glass blocks. Two additional scintillator paddles $\left(60 \times 85 \mathrm{~mm}^{2}\right)$ were placed in a cross configuration at beam entrance and one larger paddle was placed at beam exit.

A prototype of the ToT discriminator board (with one discriminator per channel, rather than two) was tested together with A2. For much of the test, our board was used together with commercial QDCs and TDCs, but in dedicated runs, the times were also measured with a prototype setup using 

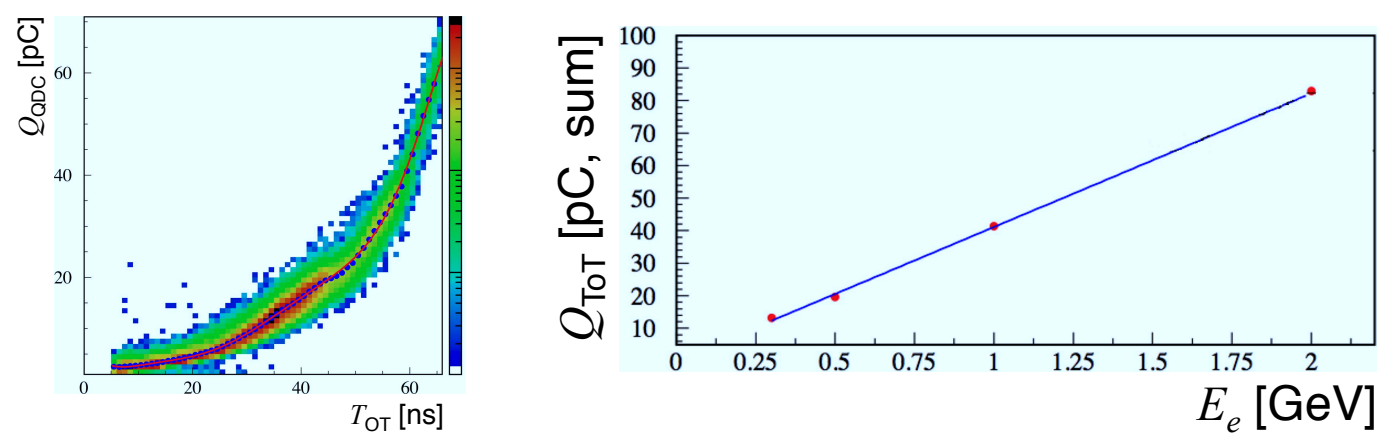

Figure 4: Left: scatter plot of signal charge measured using the QDCs vs. signal time over threshold, for electrons of various energies at the T9 test beam. Right: equivalent charge-integrated signal from ToT vs. incident electron energy, demonstrating linearity of response.
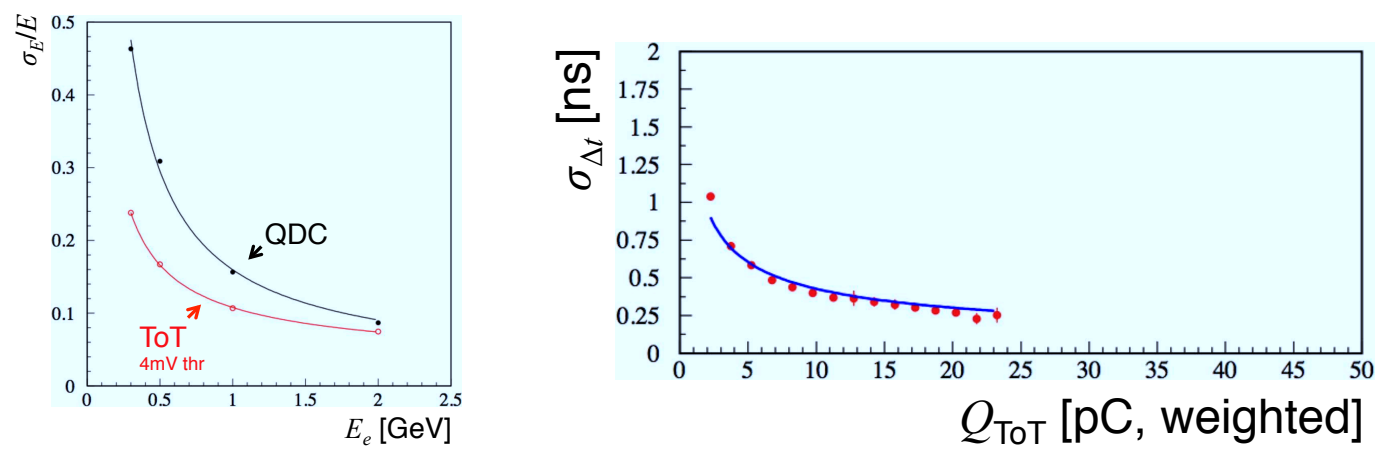

Figure 5: Left: energy resolution obtained using the ToT technique compared with that obtained using the QDCs, as a function of incident electron energy. Right: time resolution obtained using the ToT technique as a function of incident electron energy.

a TELL1 board from LHCb and an early version of the TDC mezzanine. Figure 4 (left) shows a scatter plot of the signal charge measured using the QDCs vs ToT of $4 \mathrm{mV}$, for electrons. The data are summed for all blocks on which the beam was incident and over runs of different energies.

Parameterizations of the type illustrated in 4 (left) are used to convert the time over threshold to an effective charge measurement. Figure 4 (right) demonstrates that good linearity of response is obtained using this method. The plot shows the equivalent charge in $\mathrm{pC}$ from the ToT measurement (with $4 \mathrm{mV}$ threshold) for electrons of energy $0.3,0.5,1$, and $2 \mathrm{GeV}$. Linearity to within $1 \%$ is observed. The energy resolution obtained using the ToT technique is compared with that obtained using the QDCs in 5 (left). As expected from the form of the curve in 4 (left), at low energies, the resolution obtained with the ToT technique is better than that obtained with the QDCs. The fits in 5 (left) give

$$
\begin{aligned}
\text { QDC : } & \sigma_{E} / E=8.6 \% / \sqrt{E[\mathrm{GeV}]} \oplus 13 \% / E, \\
\text { ToT : } & \sigma_{E} / E=9.2 \% / \sqrt{E[\mathrm{GeV}]} \oplus 5 \% / E \oplus 2.5 \% .
\end{aligned}
$$

While, as expected, the statistical contribution to the energy resolution is about the same with either readout scheme, the contribution from noise (term proportional to $1 / E$ ) appears to be significantly smaller with the ToT technique. The presence of the constant term with the ToT technique may be 
due to small differences in the charge vs. ToT curves from block to block, and if so, can potentially be reduced.

To study the time resolution, it is first necessary to correct for slewing. The event time reference is obtained from the scintillator paddles and Cerenkov counters. A fit to the measurements of $\sigma_{t}$ vs. charge from ToT ( $4 \mathrm{mV}$ threshold) for a single block gives $\sigma_{t}=220 \mathrm{ps} / \sqrt{E[\mathrm{GeV}]} \oplus 140 \mathrm{ps}$, where the constant term is assumed to be due to trigger jitter. Assuming that two blocks have the same intrinsic time response and that there is no common-mode contribution to the resolution, $\sigma_{t_{1}}=p / \sqrt{E_{1}}$ and $\sigma_{t_{2}}=p / \sqrt{E_{2}}$, so that we expect $\sigma_{\Delta t}=p /\left[E_{1} E_{2} /\left(E_{1}+E_{2}\right)\right]^{1 / 2}$, with no constant term. Figure 5 (right) shows the measurements of $\sigma_{\Delta_{t}}$ in slices of the weighted charge measurements from ToT, $Q=Q_{1} Q_{2} /\left(Q_{1}+Q_{2}\right)$ (4 mV threshold). The overlaid curve has $p=210 \mathrm{ps}$ and no constant term, which is indicative of the intrinsic time resolution of the detector.

\section{Acknowledgments}

We warmly thank C. Capoccia and A. Cecchetti of the Experimental Apparatus Design Service (SPAS) at the INFN Frascati Laboratories, and S. Bianucci of INFN Pisa, for their collaboration on the mechanical design of the LAV system, including the steel vacuum vessels. We also thank our mechanical design team for their assistance in oversight of the construction of the steel vessels and of the transport of the completed detectors to CERN. We thank G. Corradi, D. Tagnani, and C. Paglia of the Electronics Service (SELF) at INFN Frascati for their collaboration on the design of the new HV dividers and front-end electronics boards. Construction was made possible by the contributions from our INFN technicians: E. Capitolo, R. Lenci, V. Russo, M. Santoni, T. Vassilieva, and S. Valeri (Frascati); F. Cassese and L. Roscilli (Napoli); L. Berretta and G. Petragnani (Pisa); and F. Pellegrino (Roma). Finally, We thank V. Lollo and P. Chimenti of the Vacuum Service at INFN Frascati for their assistance with vacuum-related issues.

\section{References}

[1] V. Cirigliano et al., 2011, in press, arXiv:1107.6001.

[2] E949 Collaboration, A. Artamonov, et al., Phys. Rev. Lett., vol. 101, p. 191802, 2008.

[3] F. Hahn (ed.) et al., "NA62 technical design document," 2010, NA62 Document 10-07.

[4] OPAL Collaboration, K. Ahmet, et al., Nucl. Instrum. Meth. A, vol. 305, p. 275, 1991.

[5] F. Ambrosino et al., in 2007 IEEE Nuclear Science Symposium Conf. Record, Honolulu, Oct. 2007, pp. N05-6, arXiv:0711.3398.

[6] A. Antonelli et al., "The NA62 LAV front-end electronics," 2011, in press.

[7] B. Angelucci et al., in 2011 IEEE Nuclear Science Symposium Conf. Record, Valencia, Spain, Oct. 2011, pp. NP2.S-105. 